\title{
Modeling the Electrostatic Deflection of a MEMS Multilayers Based Actuator
}

\author{
Hassen M. Ouakad, Mohammad A. Hawwa, and Hussain M. Al-Qahtani \\ Department of Mechanical Engineering, King Fahd University of Petroleum \& Minerals, Dhahran 31261, Saudi Arabia \\ Correspondence should be addressed to Hassen M. Ouakad; houakad@kfupm.edu.sa
}

Received 2 July 2013; Revised 26 October 2013; Accepted 1 November 2013

Academic Editor: Wei-Chiang Hong

Copyright (C) 2013 Hassen M. Ouakad et al. This is an open access article distributed under the Creative Commons Attribution License, which permits unrestricted use, distribution, and reproduction in any medium, provided the original work is properly cited.

\begin{abstract}
An actuator comprised of a rigid substrate and two parallel clamped-clamped microbeams is modeled under the influence of electrostatic loading. The problem is considered under the context of nonlinear Euler's mechanics, where the actuating system is described by coupled integrodifferential equations with relevant boundary conditions. Galerkin-based discretization is utilized to obtain a reduced-order model, which is solved numerically. Actuators with different gap sizes between electrode and beams are investigated. The obtained results are compared to simulations gotten by the finite-element commercial software ANSYS.
\end{abstract}

\section{Introduction}

One of the basic and most common MEMS devices is the parallel-plate electrostatic actuator. A clear advantage of the parallel-plate electrostatic actuators is their capability of generating high force. One drawback of these actuators, however, is the low deflection they can perform due to the gap size between the parallel plates and the induced pull-in instability caused by system nonlinearities. The main source of these nonlinearities is the fact that the electrostatic produced force is inversely proportional to the squared value of the gap distance between the two electrodes. Inclusive analyses of the pull-in instability can be found in published papers by Gupta et al. [1], Nielson and Barbastathis [2], Nayfeh et al. [3], and Khater et al. [4].

In order to put limitations on the instability domain, an intuitive solution is to decrease the gap distance between the parallel plates. Two additional actions can also help: (i) reduction of the rigidity of the parallel plates and (ii) increase the areas of the electrostatic surfaces. There have been several attempts to increase the stable range of travel of parallel-plate actuators. Early attempts include the use of curved electrodes [5], utilizing leveraged bending and strain stiffening [6], and employing feedback control algorithms to increase dynamic range [7].
A unique approach of intensifying the electrostatic force and increasing the out-of-plane deflection was to utilize more than one parallel-plate electrostatic device built in parallel fashion. Abbaspour-Sani and Afrang [8] proposed a structure composed of two displaceable microplates for a microswitch application. Chaffey and Austin [9] presented another microswitch made of a double-cantilever microbeam structure. Samaali et al. [10] demonstrated a double-cantilever microbeam to design an RF microswitch. In all of these attempts, researchers were able to reduce pullin voltages, switching time and power consumption.

Inspired by these works, the current work is intended to provide an analytical solution for the deflection of a parallel double actuator comprised of a fixed electrostatic substrate and two electrostatic parallel layers. A continuum model based on Euler's beam is used to describe the two clampedclamped parallel microbeams under the influence of applied voltage. The presented model includes the effect of geometric nonlinearity due to midplane stretching. The quasi-static response of a single actuator (made of a substrate and a single electrostatic layer) is compared with that of double actuators (made of a substrate and two electrostatic layers). Then, a finite-element numerical solution is obtained by utilizing ANSYS, and a comparison between both solutions is presented. 


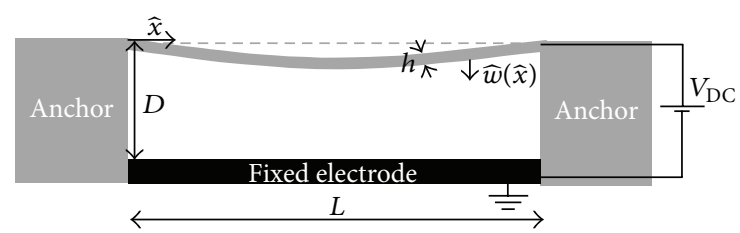

FIGURE 1: Schematic of an electrostatically actuated clampedclamped single microbeam.

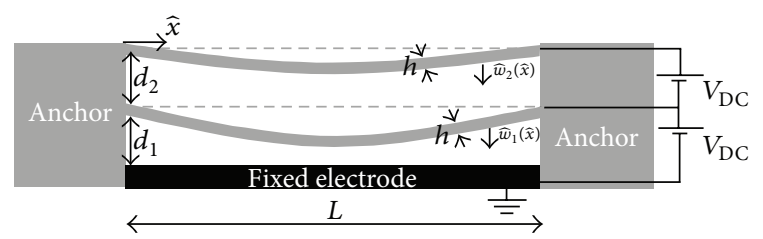

FIGURE 2: Schematic of electrostatically actuated clamped-clamped doublemicrobeams.

\section{Electromechanical Model}

In this section, we present the structural-electrical model of single-microbeam as well as double-microbeam actuators under electrostatic loading due to parallel-plate effect. Figure 1 shows a clamped-clamped single-microbeam actuator loaded by a DC electric load. The initial distance separating the microbeam from the substrate is $D$. The microbeam is modeled as a linear prismatic Euler-Bernoulli beam of width $b$, thickness $h$, length $L$, elasticity modulus $E$, density $\rho$, crosssection area $A=b h$, and area moment of inertia $I=b h^{3} / 12$. It is assumed that the microbeam is not subjected to any induced axial loads (due to residual stresses). We let $\widehat{w}(\widehat{x})$ be the beam displacement at location $\widehat{x}$.

Based on the above assumptions, the equation of motion and associated boundary conditions of the microbeam shown in Figure 2 can be expressed as in Ijntema and Tilmans [11] as follows:

$$
\begin{aligned}
E I \frac{d^{4} \widehat{w}}{d \widehat{x}^{4}} & =\left[\frac{E A}{2 L} \int_{0}^{L}\left(\frac{d \widehat{w}}{d \widehat{x}}\right)^{2} d \widehat{x}\right] \frac{d^{2} \widehat{w}}{d \widehat{x}^{2}}+\frac{\varepsilon_{0} b V_{\mathrm{DC}}^{2}}{2(D-\widehat{w})^{2}} \\
\widehat{w}(0) & =\widehat{w}(L)=0, \quad \frac{d \widehat{w}}{d \widehat{x}}(0)=\frac{d \widehat{w}}{d \widehat{x}}(L)=0 .
\end{aligned}
$$

Figure 2 shows a clamped-clamped electrically actuated double-microbeam actuator. The nonlinear equations of the system and associated boundary conditions can be described as follows [11, 12]:

$$
\begin{gathered}
E I \frac{d^{4} \widehat{w}_{1}}{d \widehat{x}^{4}}=\left[\frac{E A}{2 L} \int_{0}^{L}\left(\frac{d \widehat{w}}{d \widehat{x}}\right)^{2} d \widehat{x}\right] \frac{d^{2} \widehat{w}_{1}}{d \widehat{x}^{2}} \\
+\frac{\varepsilon_{0} b V_{\mathrm{DC}}^{2}}{2\left(d_{1}-\widehat{w}_{1}\right)^{2}}+\frac{\varepsilon_{0} b V_{\mathrm{DC}}^{2}}{2\left(d_{2}-\widehat{w}_{1}-\widehat{w}_{2}\right)^{2}}, \\
E I \frac{d^{4} \widehat{w}_{2}}{d \widehat{x}^{4}}=\left[\frac{E A}{2 L} \int_{0}^{L}\left(\frac{d \widehat{w}_{2}}{d \widehat{x}}\right)^{2} d \widehat{x}\right] \frac{d^{2} \widehat{w}_{2}}{d \widehat{x}^{2}}+\frac{\varepsilon_{0} b V_{\mathrm{DC}}^{2}}{2\left(d_{2}-\widehat{w}_{1}-\widehat{w}_{2}\right)^{2}}, \\
\widehat{w}_{1}(0)=\widehat{w}_{1}(L)=0, \quad \frac{d \widehat{w}_{1}}{d \widehat{x}}(0)=\frac{d \widehat{w}_{1}}{d \widehat{x}}(L)=0, \\
\widehat{w}_{2}(0)=\widehat{w}_{2}(L)=0, \quad \frac{d \widehat{w}_{2}}{d \widehat{x}}(0)=\frac{d \widehat{w}_{2}}{d \widehat{x}}(L)=0,
\end{gathered}
$$

where $\widehat{w}_{i}$ is the static deflection of each microbeam, $\rho$ is the mass density, $b$ and $h$ are, respectively, the width and height of the beam section, $L$ is the beam length, $E$ is Young's modulus, $A=b h$ is the cross-section area, $I=$ $b h^{3} / 12$ is the second moment of area, $\epsilon_{0}$ is the dielectric constant, and $V_{\mathrm{DC}}$ is the applied DC voltage. We note that the microbeam static behavior depends on four factors: (i) microbeam resistance to bending, (ii) microbeam stiffness due to midplane stretching, (iii) external force input (the electrostatic force term), which was derived in assuming parallel-plate theory, and (iv) overlap between the areas of each microbeam and the stationary electrode.

For convenience, we introduce the following nondimensional variables:

$$
w=\frac{\widehat{w}}{D}, \quad x=\frac{\widehat{x}}{L}, \quad w_{1}=\frac{\widehat{w}_{1}}{d_{1}}, \quad w_{2}=\frac{\widehat{w}_{2}}{d_{1}} .
$$

In nondimensional forms, (1)-(2) become

$$
\begin{aligned}
\frac{d^{4} w}{d x^{4}} & =\alpha_{11}\left[\int_{0}^{L}\left(\frac{d w}{d x}\right)^{2} d x\right] \frac{d^{2} w}{d x^{2}}+\frac{\alpha_{21} V_{\mathrm{DC}}^{2}}{(1-w)^{2}}, \\
w(0) & =w(1)=0, \quad \frac{d w}{d x}(0)=\frac{d w}{d x}(1)=0, \\
\frac{d^{4} w_{1}}{d x^{4}} & =\alpha_{12}\left[\int_{0}^{L}\left(\frac{d w_{1}}{d x}\right)^{2} d x\right] \frac{d^{2} w_{1}}{d x^{2}}+\frac{\alpha_{22} V_{\mathrm{DC}}^{2}}{\left(1-w_{1}\right)^{2}} \\
& +\frac{\alpha_{22} V_{\mathrm{DC}}^{2}}{\left(\left(d_{2} / d_{1}\right)-w_{1}-w_{2}\right)^{2}},
\end{aligned}
$$




$$
\begin{gathered}
\frac{d^{4} w_{2}}{d x^{4}}=\alpha_{12}\left[\int_{0}^{L}\left(\frac{d w_{2}}{d x}\right)^{2} d x\right] \frac{d^{2} w_{2}}{d x^{2}} \\
+\frac{\alpha_{22} V_{\mathrm{DC}}^{2}}{\left(\left(d_{2} / d_{1}\right)-w_{1}-w_{2}\right)^{2}}, \\
w_{1}(0)=w_{1}(1)=0, \quad \frac{d w_{1}}{d \hat{x}}(0)=\frac{d w_{1}}{d \hat{x}}(1)=0, \\
w_{2}(0)=w_{2}(1)=0, \quad \frac{d w_{2}}{d \widehat{x}}(0)=\frac{d w_{2}}{d \widehat{x}}(1)=0, \\
\alpha_{11}=6\left(\frac{D}{h}\right)^{2}, \quad \alpha_{12}=6\left(\frac{d_{1}}{h}\right)^{2}, \\
\alpha_{21}=\frac{6 \varepsilon_{0} L^{4}}{E^{\prime} D^{3} h^{3}}, \quad \alpha_{22}=\frac{6 \varepsilon_{0} L^{4}}{E^{\prime} d_{1}^{3} h^{3}} .
\end{gathered}
$$

Equations (4) and (5) are discretized using a Galerkin procedure to yield a reduced-order model (ROM) [13]. First, (4) is multiplied by $(1-w)^{2}$. Then, we approximate the microbeam deflection as

$$
w(x)=\sum_{i=1}^{n} a_{i} \phi_{i}(x),
$$

where $\phi_{i}(x)(i=1,2, \ldots, n)$ are the normalized mode shapes of clamped-clamped microbeam and $a_{i}(i=1,2, \ldots, n)$ are non-time-varying constant coefficients. Because of the symmetric nature of a clamped-clamped microbeam and the electrostatic forces, only symmetric (odd) mode shapes are considered.

After substituting (9) into the resulting equation, multiplying by $\phi_{i}(x)$, using the orthogonality conditions of the mode shapes, and integrating numerically the outcome from 0 to 1 , as shown below in (12), yield the following ROM equations:

$$
\begin{gathered}
\int_{0}^{1}\left\{\left[1-\sum_{i=1}^{n} a_{i} \phi_{i}(x)\right]\left[\sum_{i=1}^{n} u_{i}(t) \phi_{i}^{i v}(x)\right] \phi_{j}(x) d x\right\} \\
=\alpha_{2} V_{\mathrm{DC}}^{2} \int_{0}^{L} \phi_{j}(x) d x+\alpha_{12}\left(\int_{0}^{1}\left[\sum_{i=1}^{n} a_{i} \phi_{i}^{\prime}(x)\right]^{2} d x\right) \\
\times\left(\int _ { 0 } ^ { L } \left\{\left[1-\sum_{i=1}^{n} a_{i} \phi_{i}(x)\right]\right.\right. \\
\left.\left.\times\left[\sum_{i=1}^{n} a_{i} \phi_{i}^{\prime \prime}(x)\right] \phi_{j}(x) d x\right\}\right), \\
j=1, \ldots, n .
\end{gathered}
$$

The obtained ROM contains a system of nonlinear algebraic equations that can be solved numerically using NewtonRaphson method to obtain the non-time-varying constant coefficients $a_{i}$ and, hence, the static deflection of the singlemicrobeam actuator.
The same procedure described in the above paragraph is applied to system of two equations describing the deflection of each microbeam in the double-microbeam actuator system. First, (6) is multiplied by $\left(1-w_{1}\right)^{2}\left(\left(d_{2} / d_{1}\right)-w_{1}-w_{2}\right)^{2}$; then, each beam deflection $w_{i}(i=1,2)$ is approximated, respectively, as

$$
\begin{aligned}
& w_{1}(x)=\sum_{i=1}^{n} u_{i} \phi_{i}(x), \\
& w_{2}(x)=\sum_{i=1}^{m} \xi_{i} \phi_{i}(x),
\end{aligned}
$$

where $\phi_{i}(x)$ are the normalized mode shapes of clampedclamped microbeam and $u_{i}$ and $\xi_{i}$ are the nondimensional non-time-varying constant coefficients of the first and second beams, respectively.

At this point, we substitute (11) into (6) and (7), multiply by $\phi_{i}(x)$, use the orthogonality conditions of the mode shapes, and, then, integrate the outcome from 0 to 1 to obtain the ROM of the double-microbeam actuator.

Equations (12) below form a system of nonlinear algebraic equations in terms of these coefficients $u_{i}$ and $\xi_{i}$. These equations are solved numerically using the Newton-Raphson method to obtain the non-time-varying constant coefficients and, hence, the static deflection of the double-microbeam actuator. Consider the following:

$$
\begin{aligned}
& \int_{0}^{L}\left\{\left[\frac{d_{2}}{d_{1}}-\sum_{i=1}^{n} u_{i} \phi_{i}(x)-\sum_{i=1}^{m} \xi_{i} \phi_{i}(x)\right]\right. \\
& \left.\quad \times\left[1-\sum_{i=1}^{n} u_{i} \phi_{i}(x)\right]\left[\sum_{i=1}^{n} u_{i} \phi_{i}^{i v}(x)\right] \phi_{j}(x) d x\right\} \\
& =\alpha_{22} V_{\mathrm{DC}}^{2} \int_{0}^{L} \phi_{j}(x)\left[\frac{d_{2}}{d_{1}}-\sum_{i=1}^{n} u_{i} \phi_{i}(x)-\sum_{i=1}^{m} \xi_{i} \phi_{i}(x)\right] d x \\
& +\alpha_{22} V_{\mathrm{DC}}^{2} \int_{0}^{L} \phi_{j}(x)\left[1-\sum_{i=1}^{n} u_{i} \phi_{i}(x)\right] d x \\
& +\alpha_{12}\left(\int_{0}^{L}\left\{\left[\sum_{i=1}^{n} u_{i} \phi_{i}^{\prime}(x)\right]^{2}\right\} d x\right) \\
& \quad \times\left(\int _ { 0 } ^ { 1 } \left\{\left[\frac{d_{2}}{d_{1}}-\sum_{i=1}^{n} u_{i} \phi_{i}(x)-\sum_{i=1}^{m} \xi_{i} \phi_{i}(x)\right]\right.\right. \\
& \left.\left.\quad \times\left[\sum_{i=1}^{n} u_{i} \phi_{i}^{\prime \prime}(x)\right] \phi_{j}(x) d x\right\}\right), \\
&
\end{aligned}
$$




$$
\begin{aligned}
& \int_{0}^{L}\left\{\left[\frac{d_{2}}{d_{1}}-\sum_{i=1}^{n} u_{i} \phi_{i}(x)-\sum_{i=1}^{m} \xi_{i} \phi_{i}(x)\right]\right. \\
& \left.\quad \times\left[\sum_{i=1}^{m} \xi_{i} \phi_{i}^{i v}(x)\right] \phi_{j}(x) d x\right\} \\
& =\alpha_{22} V_{\mathrm{DC}}^{2} \int_{0}^{L} \phi_{j}(x)\left[\frac{d_{2}}{d_{1}}-\sum_{i=1}^{n} u_{i} \phi_{i}(x)-\sum_{i=1}^{m} \xi_{i} \phi_{i}(x)\right] d x \\
& \quad+\alpha_{12}\left(\int_{0}^{L}\left\{\left[\sum_{i=1}^{m} \xi_{i} \phi_{i}^{\prime}(x)\right]^{2}\right\} d x\right) \\
& \quad \times\left(\int _ { 0 } ^ { L } \left\{\left[\frac{d_{2}}{d_{1}}-\sum_{i=1}^{n} u_{i} \phi_{i}(x)-\sum_{i=1}^{m} \xi_{i} \phi_{i}(x)\right]\right.\right. \\
& \left.\left.\quad \times\left[\sum_{i=1}^{m} \xi_{i} \phi_{i}^{\prime \prime}(x)\right] \phi_{j}(x) d x\right\}\right)
\end{aligned}
$$

\section{Finite-Element Method}

The commercial software ANSYS is used to model the singleand double-beam configuration. Two and four layers are meshed using standard tetrahedral elements. The convergence of the solution has been examined, and a sufficiently fine mesh has been used. Figure 3 shows the ANSYS doublemicrobeam actuator model performed in this work. The ANSYS model consists of a coupled electrostatic-structural element (TRANS126 elements) to model the electrostatic coupling between the beam and a ground electrode. This element is a two-node element which has one structural degree of freedom and an electrical potential between the nodes. One end of each element is held fixed, while the other is coupled to a structural node in the beam. A voltage difference is applied across the TRANS126 element, which creates an attractive force that is resisted by the stiffness of the beam. The comparison shows excellent agreement among the ROM nonlinear beam model and the nonlinear finiteelement model.

\section{Results and Discussion}

Representative cases are presented in the sequel for a singlemicrobeam actuator and a double-microbeam actuator. Let us assume that each microbeam has the geometric properties of $L=150 \mu \mathrm{m}, h=1 \mu \mathrm{m}$, and $b=4 \mu \mathrm{m}$, and the gap thicknesses are governed by $D=d_{1}+d_{2}+h=3 \mu \mathrm{m}$.

In order to ensure the convergence of the ROM, we calculate the maximum static deflection of the upper microbeam $\left(w_{\max }=\left.w_{2}\right|_{x=(1 / 2) L}\right)$ for the case of double-microbeam actuator using one, two, and three symmetric modes of the ROM while varying the DC load. Figure 4 shows that using one mode yields acceptable converged results, which

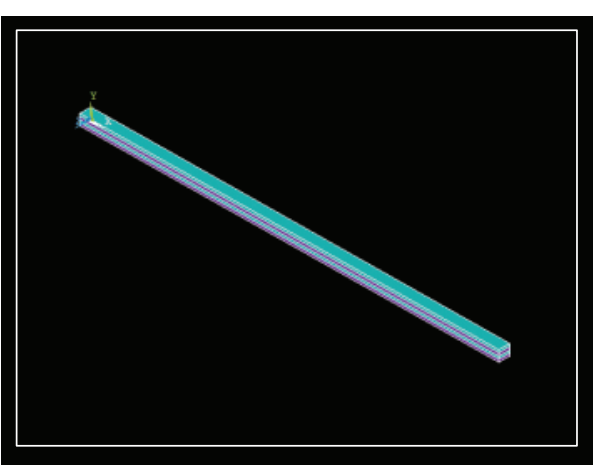

FIGURE 3: ANSYS finite-element model of double-microbeam actuator.

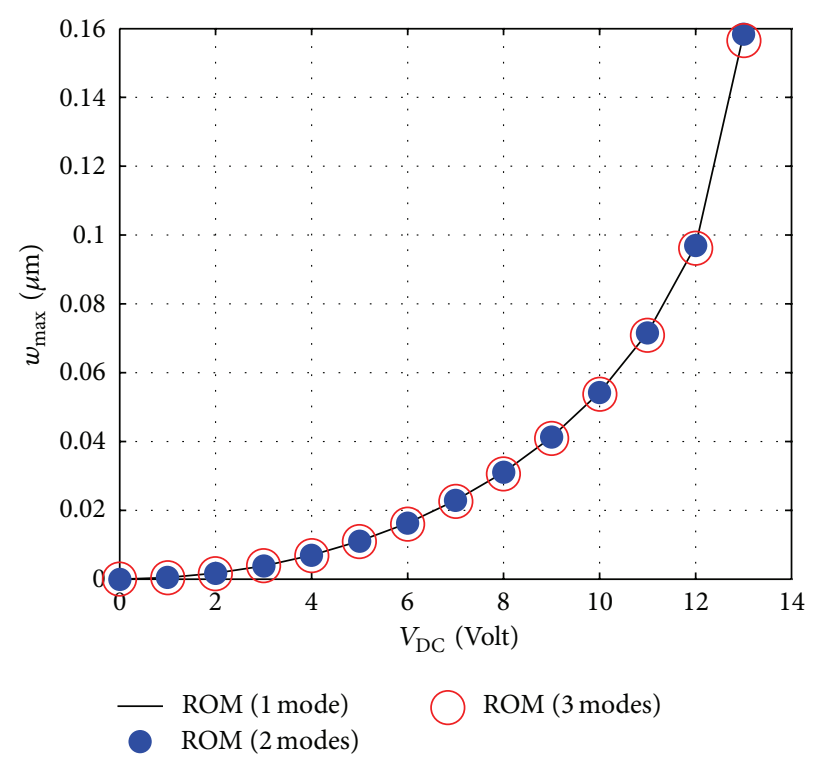

FIGURE 4: Variation of the maximum static deflection of the upper beam of the double-microbeam actuator for different applied DC voltages.

indicates a minimum contribution of the higher-order modes to the total outcome.

Next, we move on to compare the maximum static defection of the single-microbeam actuator with that of the doublemicrobeam actuator. Figure 5 clearly shows that the doublemicrobeam actuator is capable of attaining a larger stroke than the single-microbeam actuator, but on the expense of a lower pull-in voltage.

The analytical results obtained from the reduced-order model are then compared with the results obtained from finite element ANSYS simulation. Figure 6 shows a good agreement between the two approaches, with slight deviations observed at higher voltage loads.

Hence, ANSYS can be utilized to predict response of a triple-microbeams actuator. Keeping the same total gap thickness, Figure 7 shows that there is a trend of increasing maximum deflection as the actuator embraces more microbeam. It is obvious that adding more layers increases 


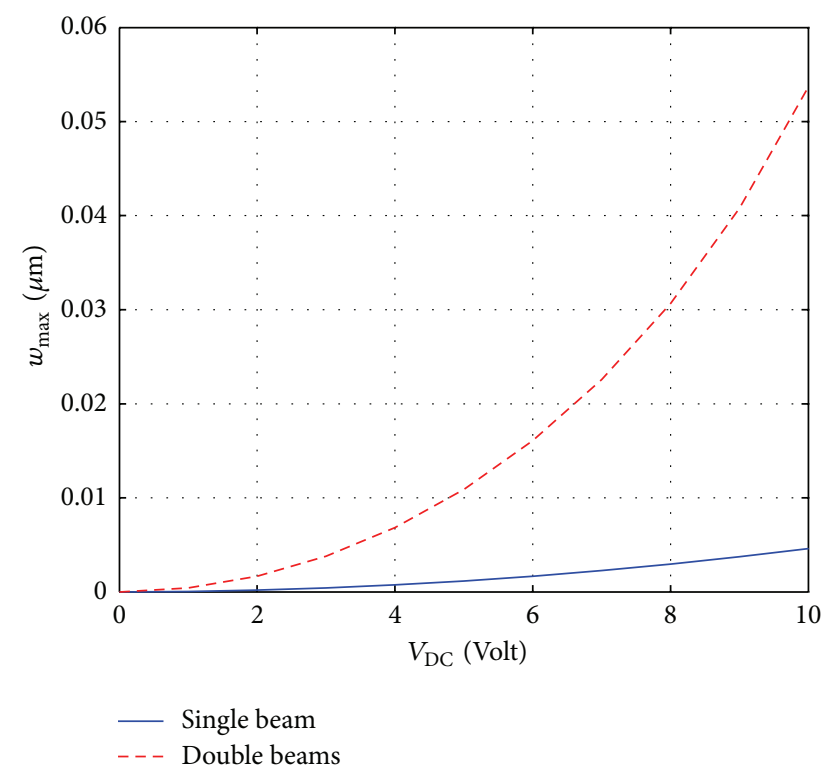

FIGURE 5: Comparison of the static response of the singlemicrobeam actuator and the upper beam of the double-microbeam actuator. The pull-in voltage values for single and doublemicrobeams are 18 and 168 Volts, respectively.

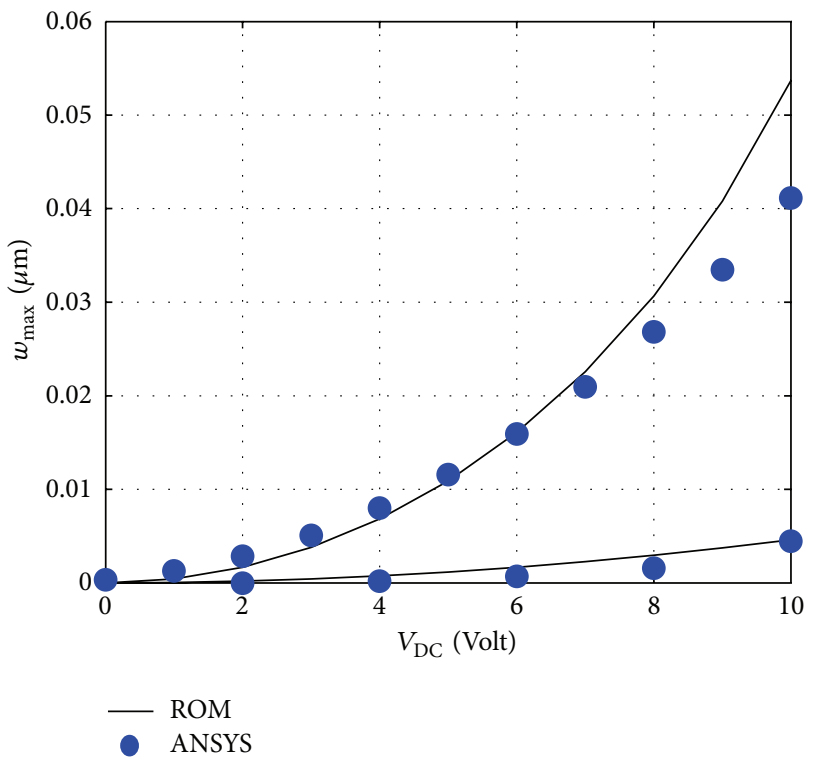

FIgURE 6: Comparison between ROM results and ANSYS FEM results.

the magnitude of the deflection albeit reducing the pull-in voltage.

Finally, we investigate the effect varying the gap thicknesses on the maximum static deflection of the doublemicrobeam- actuator. Figure 8 shows clearly that decreasing the gap between the two microbeams results in decreasing the pull-in voltage as well as increasing the range of travel (stroke) of the actuating system.

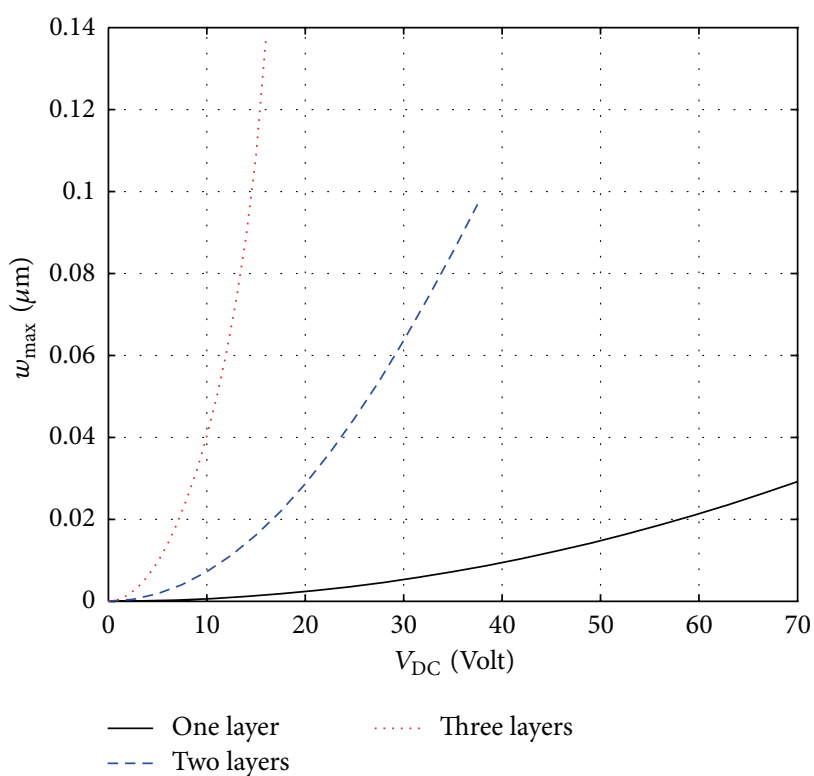

FIGURE 7: Maximum deflection versus applied DC voltage for different number of beam-electrode configuration.

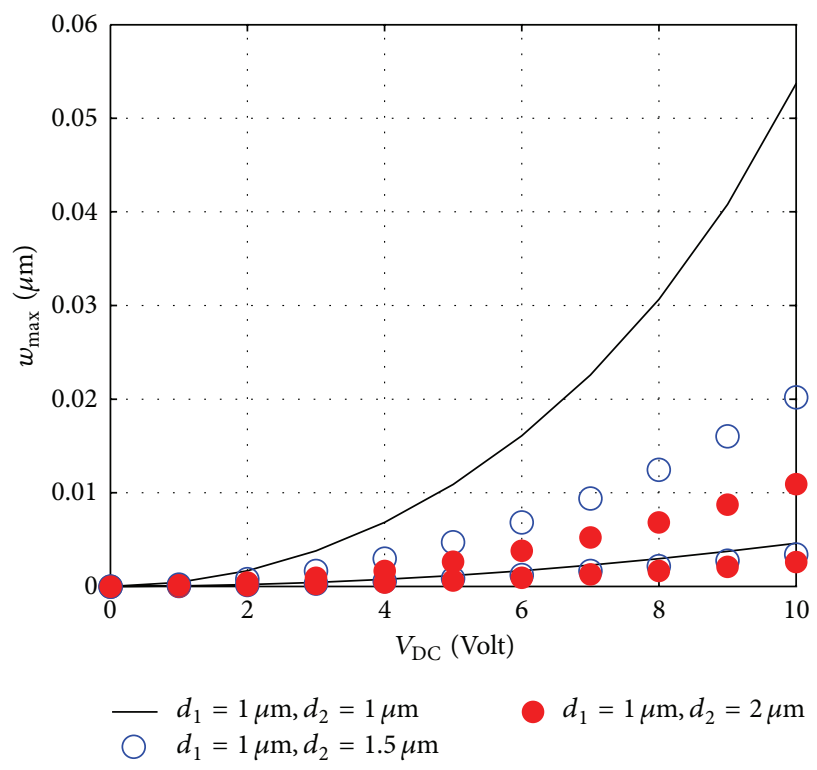

FIGURE 8: Effect of the gap sizes on the static response of the singlemicrobeam and double-microbeam actuators.

\section{Conclusions}

The paper presented a MEMS actuator comprising of an electrostatic substrate-single microbeam and substrate-double microbeams. Different arrangements of clamped-clamped microbeam-electrode combinations were presented in this study. Reduced-order model based on Galerkin method and FEM analysis were used to calculate maximum transverse deflections and obtain pull-in voltages. It is found that it is possible to stimulate the response of the system by adding more layers of micro-beam-electrode combinations. The effects of spacing between microbeams and/or electrodes are found to be of a great importance on the transverse 
response of the system as well as on the pull-in voltage value. The investigation provides a ground for implementing the technique of using multiple layers of microbeam-electrode combination whenever larger response is desired.

\section{Acknowledgment}

The authors greatly appreciate the support received from King Fahd University of Petroleum and Minerals (KFUPM) through its Deanship of Scientific Research (DSR).

\section{References}

[1] R. K. Gupta, E. S. Hung, Y. J. Yang, G. K. Ananthasuresh, and S. D. Sentura, "Pull-in dynamics of electrostatically actuated beams," in Technical Digest Solid State Sensor and Actuator Workshop, pp. 1-2, 1996.

[2] G. N. Nielson and G. Barbastathis, "Dynamic pull-in of parallelplate and torsional electrostatic MEMS actuators," Journal of Microelectromechanical Systems, vol. 15, no. 4, pp. 811-821, 2006.

[3] A. H. Nayfeh, M. I. Younis, and E. M. Abdel-Rahman, "Dynamic pull-in phenomenon in MEMS resonators," Nonlinear Dynamics, vol. 48, no. 1-2, pp. 153-163, 2007.

[4] M. E. Khater, K. Vummidi, E. M. Abdel-Rahman, A. H. Nayfeh, and S. Raman, "Dynamic actuation methods for capacitive MEMS shunt switches," Journal of Micromechanics and Microengineering, vol. 21, no. 3, Article ID 035009, 2011.

[5] R. Legtenberg, J. Gilbert, S. D. Senturia, and M. Elwenspoek, "Electrostatic curved electrode actuators," Journal of Microelectromechanical Systems, vol. 6, no. 3, pp. 257-265, 1997.

[6] E. S. Hung and S. D. Senturia, "Extending the travel range of analog-tuned electrostatic actuators," Journal of Microelectromechanical Systems, vol. 8, no. 4, pp. 497-505, 1999.

[7] J. I. Seeger and B. E. Boser, "Dynamics and control of parallelplate actuators beyond the electrostatic instability," in Proceedings of 10th International Conference on Solid-State Sensors and Actuators (Transducers '99), pp. 474-477, Sendai, Japan, June 1999.

[8] E. Abbaspour-Sani and S. Afrang, "A low voltage MEMS structure for RF capacitive switches," Progress in Electromagnetics Research, vol. 65, pp. 157-167, 2006.

[9] J. Chaffey and M. Austin, "Analytical modelling of the electromechanical coupling of cantilever beams," in Smart Structures, Devices, and Systems, Proceedings of SPIE, pp. 86-93, December 2002.

[10] H. Samaali, F. Najar, S. Choura, A. H. Nayfeh, and M. Masmoudi, "A double microbeam MEMS ohmic switch for RFapplications with low actuation voltage," Nonlinear Dynamics, vol. 63 , no. 4, pp. 719-734, 2011.

[11] D. J. Ijntema and H. A. C. Tilmans, "Static and dynamic aspects of an air-gap capacitor," Sensors and Actuators A, vol. 35, no. 2, pp. 121-128, 1992.

[12] H. Samaali, F. Najar, S. Choura, A. H. Nayfeh, and M. Masmoudi, "Dynamic study of a capacitive mems switch with double clamped-clamped microbeams," in Proceedings of the 5th International Conference on Advances in Mechanical Engineering and Mechanics (ICAMEM '10), 2010.

[13] M. I. Younis, E. M. Abdel-Rahman, and A. Nayfeh, "A reducedorder model for electrically actuated microbeam-based MEMS," Journal of Microelectromechanical Systems, vol. 12, no. 5, pp. 672-680, 2003. 


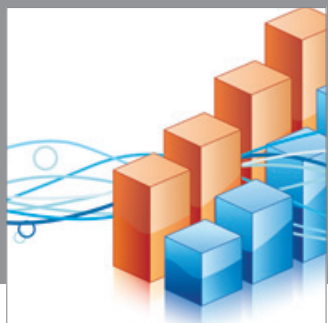

Advances in

Operations Research

mansans

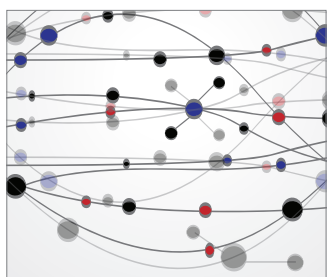

The Scientific World Journal
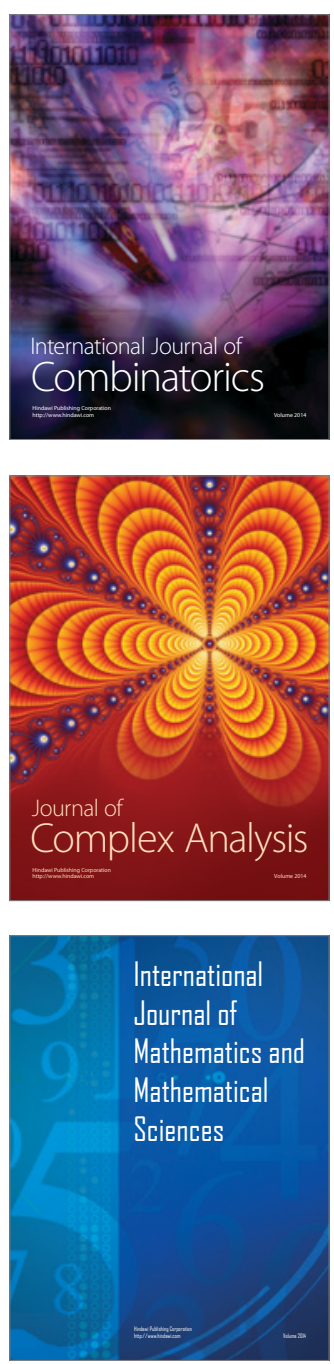
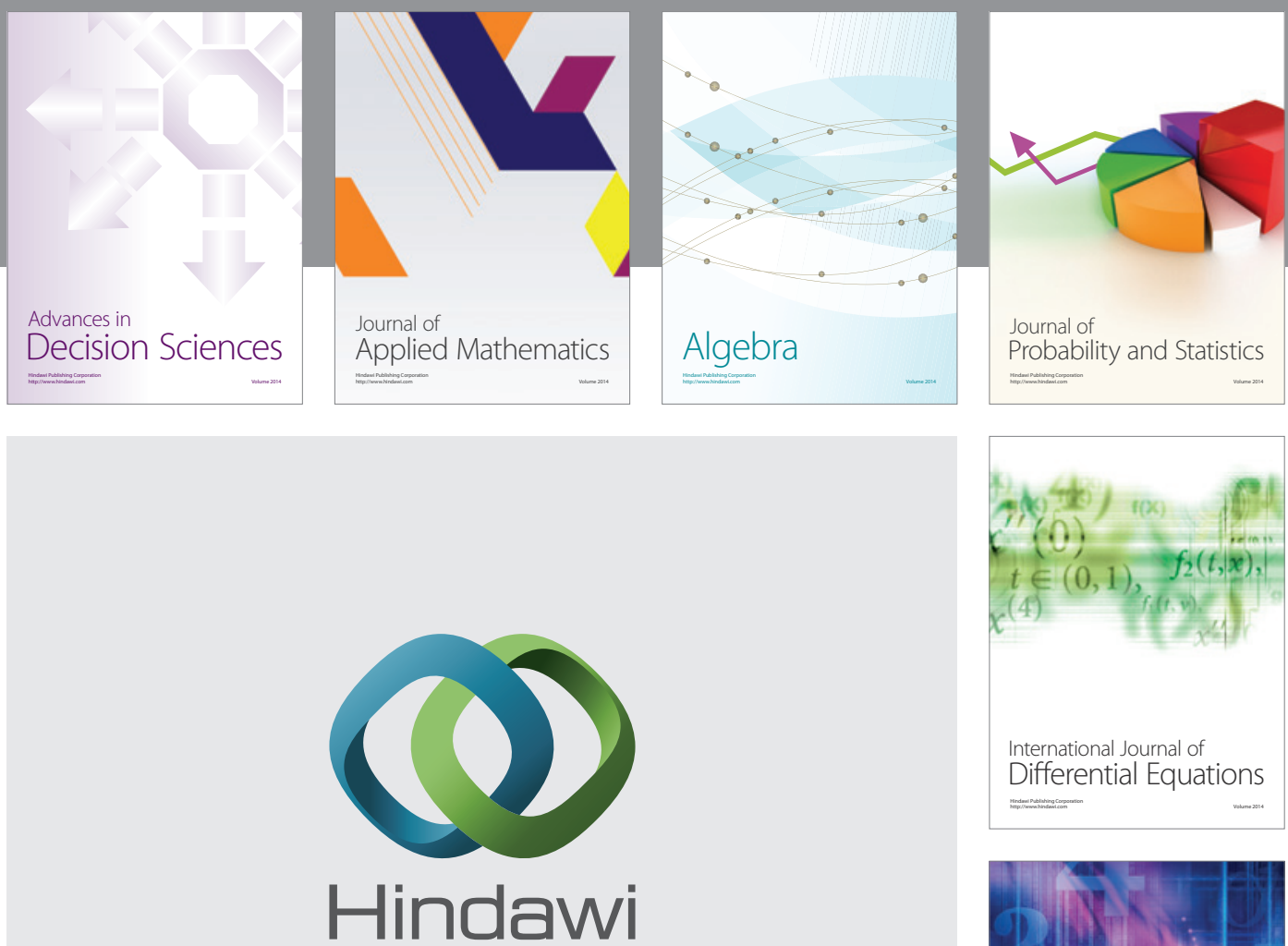

Submit your manuscripts at http://www.hindawi.com
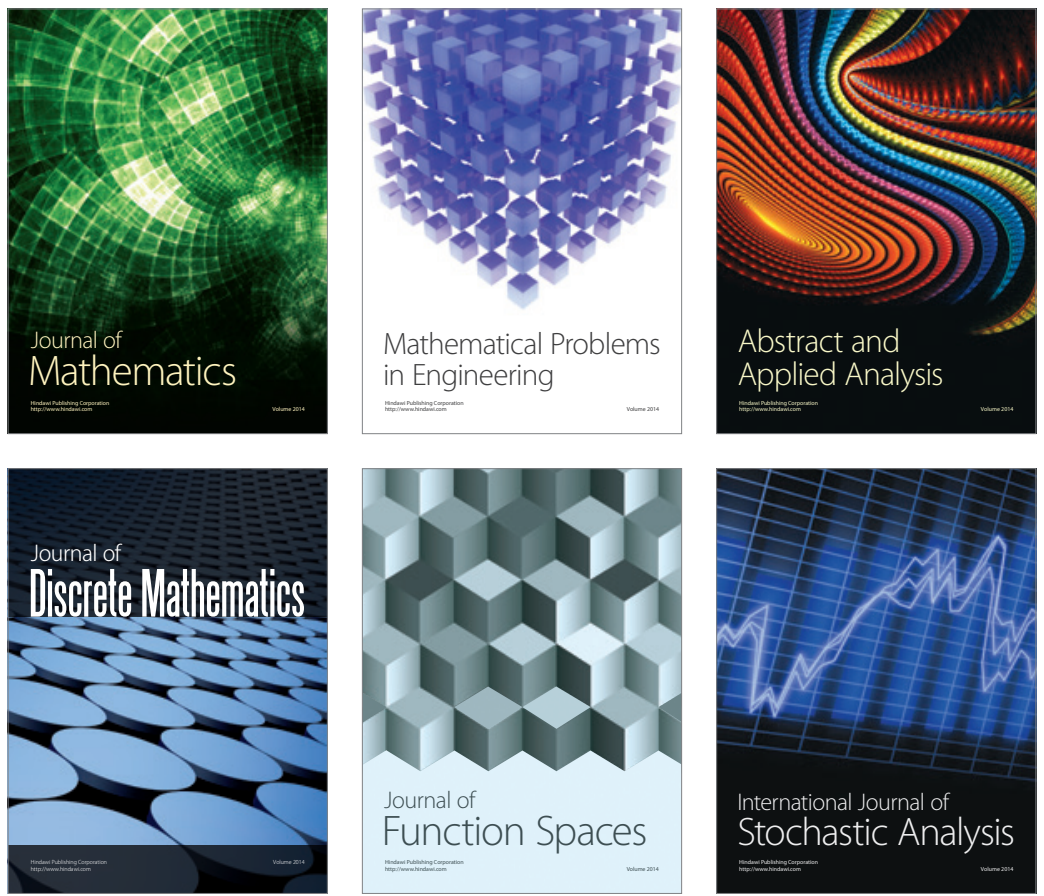

Journal of

Function Spaces

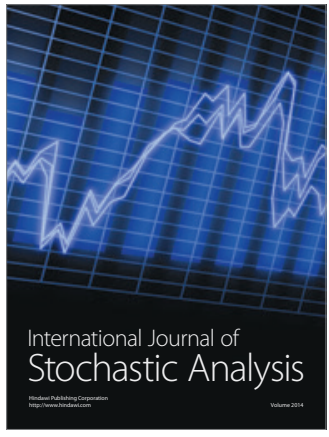

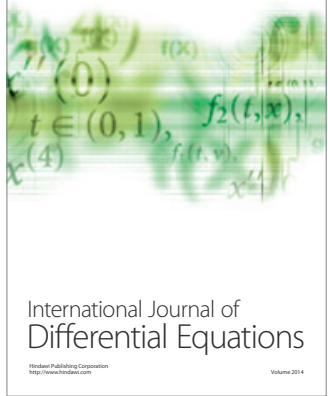
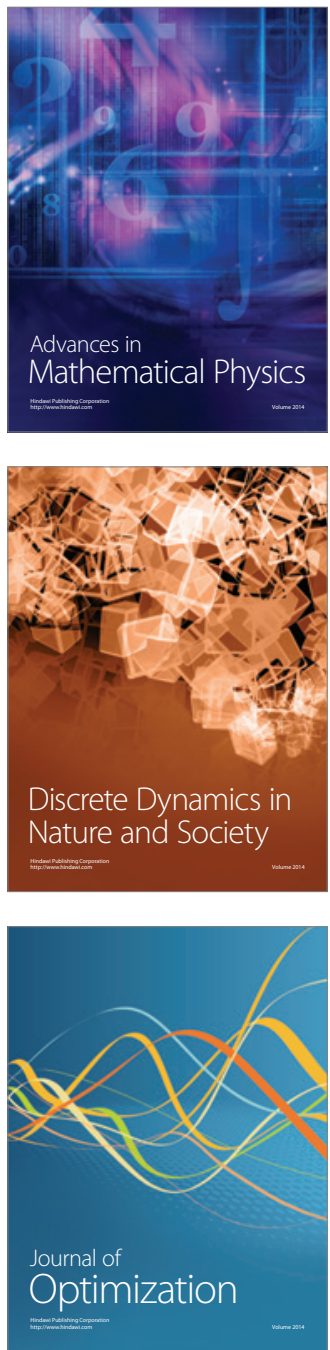